J. Clin. Chem. Clin. Biochem.

Vol. 16, 1978, pp. 365-369

\title{
Zur Umsetzung von Cyclohexylsulfaminsäure (Cyclamat) mit salpetriger Säure in wäßriger HCI- Lösung, isoliertem menschlichen Magensaft und Urin
}

Von $A$. Kinawi und $H$. Kia

Institut für Biochemie und Molekularbiologie (FB 23 / WE 03) der Freien Universität Berlin

(Eingegangen am 18. Oktober 1977/3. Februar 1978)

Zusammenfassung: Die pH-Abhängigkeit der Bildung und des Zerfalls des bei der Umsetzung der Cyclohexylsulfaminsäure mit salpetriger Säure entstehenden N-Nitrosoderivats wurde photometrisch durch kontinuierliche Messung der Absorptionsänderung untersucht. Es wurde ein optimaler pH-Wert von 2,45 ermittelt, bei dem eine maximale Ausbeute an dem zerfallenden N-Nitrosoderivat entsteht. Die Halbwertzeit $\left(t_{1 / 2}\right)$ der Zerfallsreaktion bei pH 2,61 (Molverhältnis: Cyclohexylsulfaminsäure/Natriumnitrit $=1 / 1,11$ ) liegt bei 13,6 min. Bei pH 4,5 zeigt das $\mathrm{N}$-Nitrosoderivat eine relativ hohe Stabilität.

Der Verlauf der Umsetzung in isoliertem menschlichen Urin und Magensaft zeigte die gleiche pH-Abhängigkeit wie bei der Durchführung der Umsetzung in wäßriger HCl-Lösung.

The reaction of cyclohexylsulphamic acid (Cyclamate) with nitrous acid in aqueous $\mathrm{HCl}$-solution, isolated human gastric juice and urine

Summary: The pH dependency of the formation and decomposition of the $\mathrm{N}$-nitroso derivative, which is produced by the reaction of cyclohexylsulphamic acid with nitrous acid, was investigated by means of continuous photometric measurement of the changes in absorbance. Reaction at $\mathrm{pH} 2.45$ resulted in an optimal yield of the $\mathrm{N}$-nitroso derivative. The half-life of the decomposition reaction (the reactants cyclohexylsulphamic acid/sodium nitrite being in a molar ratio of $1: 1.11$ ) at $\mathrm{pH} 2.61$ was $13.6 \mathrm{~min}$. The $\mathrm{N}$-nitroso derivative showed a relatively high stability at $\mathrm{pH} 4.5$.

The reaction in isolated human gastric juice and urine showed the same $\mathrm{pH}$ dependency as in aqueous $\mathrm{HCl}$ solution.

\section{Einleitung}

Die Süßstoffe Cyclamat und Saccharin wurden hinsichtlich einer möglichen karzinogenen, co-karzinogenen, mutagenen und cytotoxischen Wirkung untersucht $(1-10)$. Weiterhin wurden Untersuchungen über den Einfluß dieser Stoffe auf Fertilität und Lebensdauer durchgeführt (11-12). Die Ërgebnisșe dieser Untersuchungen, die an Mäusen, Ratten, Kaninchen und Rhesusaffen als Versuchstieren sowie ạn Menșchen durchgeführt wurden, êrschweren wègen häufiger $\mathrm{Ab}$ weichungen und Widersprüche eine eindeutige Stellungnahme zur Frage der Unbedenklichkeit der Anwendung von Cyclamat als Zuckerersatzmittel (13).

Während Schmähl et all. $(1,2)$ weder eine kärżinogene nöch eine co-karzinogene Wirkung dieser Süßstoffẹ nachweisen konnten, stellten Price et al. (3) das Auftreten von Blasenkrebs bei den mit Cyclamat behandelten Ver- suchstieren fest, und schließlich ist nach der Arbeit von Chowaniec et al. (4) dem Cyclamat eine co-karzinogene Wirkung zuzuschreiben.

Die unterschiedlichen Befunde von Schmähl et al. und Chowaniec et al. hinsichtlich einer möglichen Co-karzinogenität des Cyclamats sind eventuell auf die Unterschiede der angewendeten Testsysteme zurückzuführen. Während Schmähl et àl. mit dem System Cyclamat/Butyl-butanolnitrosamin das Fehlen einer co-karzinogenen Wirkung bewiesen, gelang Chowaniec et al. der Nachweis einer Co-karzinogenität mit der Kombination Cyclamat/ $\mathrm{N}$-Methyl-N-nitrosoharnstoff. Zur Erhärtung dieses Befundes weisen die Autoren darauf hin, daß das Cyclophosphamid in Kombination mit N-Methyl-N-nitrosoharnstoff keine Verstärkung der karzinogenen Wirkung des Nitrosamids bewirkt. Im Rahmen reaktionskinetischer Untersuchungen zur Entstehung von N-Nitroso- 
verbindungen aus Arzneimitteln bei Einwirkung von salpetriger Säure (14-16) wurde u. a. der Einfluß von Cyclohexylsulfaminsäure auf die Bildung von N-Nitrosoephedrin untersucht (16). Hierbei wurde die Bildung des N-Nitrosoephedrins fast vollständig gehemmt, da die Cyclohexylsulfaminsäure sich rasch mit der salpetrigen Säure umsetzt. Dieser Befund veranlaßte uns, diese Umsetzung näher zu untersuchen, zumal keine Angaben über eine mögliche Karzinogenität bzw. Co-karzinogenität von Cyclamat in Gegenwart von Nitrit bekannt sind.

Das bei der Nitrosierung von Cyclamat primär entstehende N-Nitrosoderivat zerfällt spontan unter Bildung von Schwefelsäure, Stickstoff und Carbonium-Ion, wobei das letzte sich durch Bildung von Cyclohexen und Cyclohexylnitrit stabilisiert (17-20). Im Rahmen der Erforschung einer möglichen karzinogenen bzw. co-karzinogenen Wirkung des Cyclamats ist es von Interesse, die Entstehung und den Zerfall des entsprechenden N-Nitrosoderivats in Abhängigkeit von der Nitritkonzentration sowie der Azidität des Reaktionsmediums zu untersuchen, zumal das strukturell ähnliche und ebenfalls instabile N-Nitrosoderivat des Phenacetins ein direkt wirkendes Karzinogen darstellt (21).

\section{Material und Methoden}

Natriumnitrit, Cyclohexanol, Cyclohexen, konzentrierte Salzsäure, Natriumhydroxid, Cyclohexylnitrit und Cyclohexylsulfaminsäure (p. a., Merck). Das Cyclohexylnitrit wurde nach den Angaben von Dalziel (17) hergestellt.

\section{Geräte}

1. Spektralphotometer (Beckmann-Photometer, Typ DB).

2. Schreiber (Beckmann 10" Recorder), Papiervorschub:

0,2 inch/min. Spannung: $100 \mathrm{mV}$.

3. Knick Digital-pH Meter.

Herstellung der Natriumnitrit- und Cyclohexylsulfaminsäurelösungen

1. Natriumnitritlösung: $1 \mathrm{~g} / 1$ dest. Wasser.

2. Cyclohexylsulfaminsäure-Stammlösung: $10 \mathrm{~g} / 1$ dest. Wasser.

In 100-ml-Meßkolben wurde je $1 \mathrm{ml}$ der Cyclohexylsulfaminsäure-Stammlösung mit $98 \mathrm{ml}$ dest. Wasser verdünnt, die Verdünnung mit wenig $(<1 \mathrm{ml})$ Salzsäure bzw. Natronlauge unter pH-Messung versetzt, und der Meßkolben mit dest. Wasser auf $100 \mathrm{ml}$ gefüllt. Die so erhaltenen Cyclohexylsulfaminsäurelösungen $(100 \mathrm{mg} / \mathrm{l})$ wiesen folgende $\mathrm{pH}$-Werte auf: .

1,$09 ; 1,58 ; 2,18 ; 2,61 ; 2,93 ; 3,30 ; 3,26 ; 3,42 ; 4,06$ und 5,10 .

Zur Durchführung der Umsetzung (s. u.) wurden je $1,4 \mathrm{ml}$ jeder Lösung mit 10, 20, 30, 40, $50 \mathrm{bzw} .60 \mu \mathrm{l}$ der Nitritlösung versetzt. Weiterhin wurden die in Tabelle 1 angegebenen Cyclohexylsulfaminsäure-HCl-Lösungen $(1,4 \mathrm{ml})$ mit den entsprechenden Natriumnitritverdünnungen $(60 \mu \mathrm{l})$ umgesetzt. Hierbei wurde sowohl der pH-Wert der Lösungen $(2,61)$ als auch das Molverhältnis Cyclohexylsulfaminsäure/Natriumnitrit $(1 / 1,111)$ konstant gehalten.
Tab. 1. Verdünnungsreihe der Reaktionspartner.

\begin{tabular}{lcl}
\hline $\begin{array}{l}\text { Cyclohexylsulfaminsäure- } \\
\text { HCl-Lösung (pH 2,61) mg/l }\end{array}$ & Natriumnitrit (mg/l) \\
\hline \multicolumn{1}{l}{} & 50 & 0,5 \\
1. & 25 & 0,25 \\
2. & 12,5 & 0,125 \\
3. & 6,25 & 0,0625 \\
4. & 3,125 & 0,03125 \\
5. & 1,563 & 0,01563 \\
6. & 0,781 & 0,00781 \\
7. & & \\
\hline
\end{tabular}

Um den Verlauf der Umsetzung in Magensaft und Urin zu untersuchen, wurde Cyclohexylsulfaminsäure in drei verschiedenen durch Zèntrifugation von Schleim befreiten Magensäften gelöst (50 mg/1). Die pH-Werte dieser Lösungen lagen bei 1,5,2 und 2,3. Die drei verschiedenen Urinproben $(50 \mathrm{mg} / 1$ Cyclohexylsulfaminsäure) wurden, wie oben beschrieben, durch Zusatz von wenig Natronlauge auf die pH-Werte $3,8,4,0$ und 5,1 gebracht. Je $1,4 \mathrm{ml}$ dieser Cyclohexylsulfaminsäurelösungen wurde mit je $30 \mu 1$ der Nitritlösung $(1 \mathrm{~g} / \mathrm{l})$ versetzt.

\section{Blindversuch}

Um den Einfluß der Zerfallsprodukte auf die Bestimmungsmethode zu ermitteln, wurden, unter der Annahme einer 100prozentigen Ausbeute an dem $Z$ wischenprodukt N-NitrosoCyclohexylsulfaminsäure, zu $100 \mathrm{ml}$ einer wäßrigen $\mathrm{HCl}$-Lösung (pH 2,61) jeweils $50 \mu \mathrm{l}$ Cyclohexën, Cyclohexylnitrit und Cyclohexanol zugesetzt. Die erhaltene Lösung wurde gegen Cyclohexylsulfaminsäure-HCl-Lösung (100 mg/1, pH 2,61) photometriert (320-200 nm).

\section{Durchführung}

Vorversuche zur reaktionskinetischen Untersuchung der Umsetzung der Cyclohexylsulfaminsäure mit salpetriger Säure mit Hilfe der Hochdruckflüssigkeitschromatographie haben gezeigt, daß die Úmsetzung, besonders mit żunehmender Azidität des Reaktionsmediums, zu.rasch verläuft, so daß genaue Ergebnisse nur unter kontinuierlicher Verfolgung des Reaktionsvierlaufes erzielbar sind. Aus diesem Grunde wurde die Umsetzung unter kontinuierlicher Messung der Absorption der Reaktionslösung bei $250 \mathrm{~nm}$ in einer Halbmikroküvette durchgefuhrt.

Hierzu wurden bei Raumtemperatur $\left(25^{\circ} \mathrm{C}\right)$ je $1,4 \mathrm{ml}$ der oben erwähnten Cyclohexylsulfaminsäurelösungen in eine Halbmikroküvette gegeben, mit der vorgesehenen Menge an Natriumnitritlösung versetzt, und mit Hilfe einer Pasteurpipette der Inhalt der Küvette durch zweimaliges rasches Ansaugen und Entleeren gut vermischt. Die Messung erfölgte gegen eine mit der gleichen Cyclohexylsulfaminsäurelösung gefüllte Referenzküvette. Bei der Durchführung der Umsetżung in stark saurem Bereich (pH 1 und 1,5) darf der Vorgang der Nitritzugabe und der Mischung der Reaktionslösung nicht länger als 3-4 s dauern, da sonst die Ermittlung der fựr die Auswertung benötigten Peakhöhe (s. u.) wegen Überlagerung von Bildung und Zerfall des N-Nitrosoderivats nicht möglich ist.

\section{Auswertung und Ergebnisse}

Wie der Blindversuch gezeigt hat, wird die Bestimmungsmethode durch die Entstehung der Zerfallsprodukte in der Reaktionslösung nicht gestört. Während das Absorptionsmaximum von Cyclohexylnitrit, Cyclohexen und Cyclohexanol unter den Versuchsbedingungen bei 210 $\mathrm{nm}$ liegt, zeigt das Nitrosoderivat eine relativ hohe $\mathrm{Ab}$ - 
sorption mit einem Absorptionsmaximum bei $238 \mathrm{~nm}$ (s. Abb. 1).

Da die Umsetzung, besonders in stark saurem Bereich, zu rasch verläuft, ist es nicht gelungen, die Absorptionszunahme von Beginn der Umsetzung an zu verfolgen. Es konnten die Anstiege ab pH 2,5 ermittelt werden. Aus diesem Grund wurde nicht der Anstieg der Kurven bei der Bildung des N-Nitrosoderivats als Maß für die Reaktionsgeschwindigkeit, sondern die ermittelbaren Peakhöhen (maximale Absorption) bei jeder Umsetzung verwendet (s. Abb. 2).

Als Maß für die Zerfallsgeschwindigkeit wurde der „Anstieg" $\operatorname{tg} \alpha$ der abfallenden Kurventeile verwendet (s. Abb. 2). Um die Abhängigkeit der Zerfallsgeschwindigkeit von Ausgangskonzentration und $\mathrm{pH}$-Wert zu zeigen, wurden die ermittelten tg $\alpha$-Werte auf die entsprechenden Peakhöhen bezogen (s. Tab. 2). Zur Ermittlung des pHWertes bei dem, trotz Uberlagerung der Bildungs- und der Zerfallsgeschwindigkeit, ein optimaler, für eine kurze Zeit vorhandener Anteil an N-Nitrosoderivat entsteht, wurden die ermittelten Peakhöhen gegen die entsprechenden pH-Werte aufgetragen (s. Abb. 3). Es ließ sich ein optimaler pH-Wert von 2,45 ermitteln.

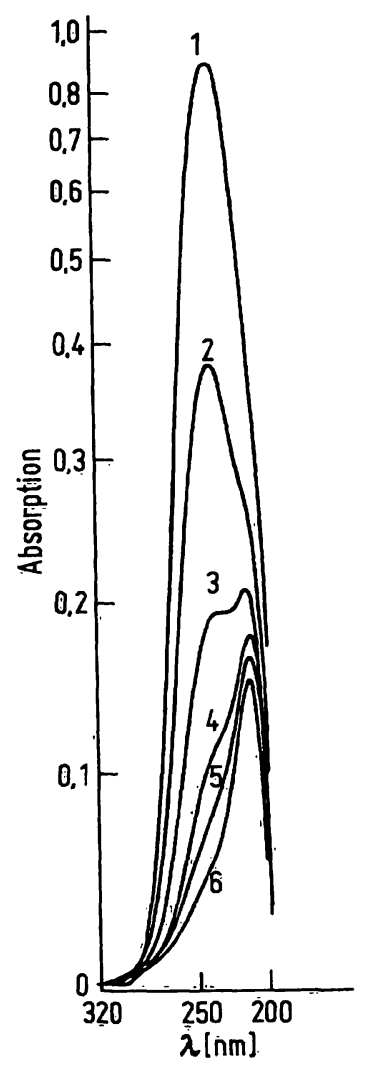

Abb. 1. UViSpektren der Reạktionnslösung unmittelbar (Spektrum 1) nach der Umsetzung von Cyclohexylsulfaminsäure mịt Natriumnitrit bei pH 2,18. Die Aufnahme der Spektren 2-6 wurde jeweils 20s nach Beendigung der vorhergehenden durchgefựhrt. Die Aufnahme jedes Spek trums dauerte $3 \mathrm{~min}$.

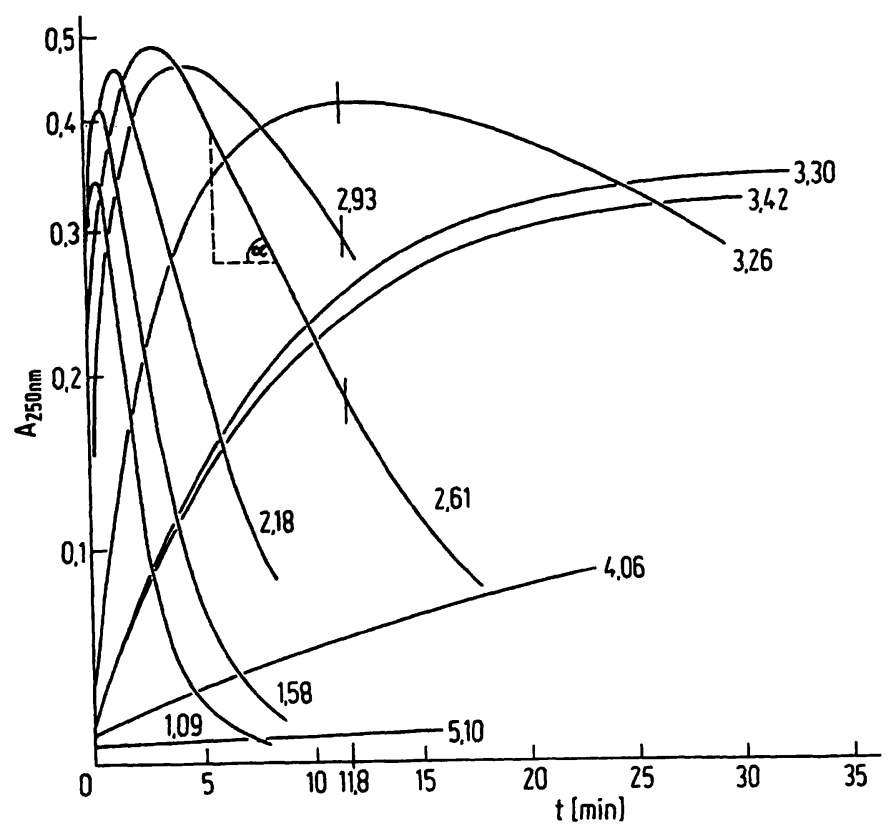

Abb. 2. Änderung der Absorption (bei $250 \mathrm{~nm}$ ) der Reaktionslösungen nach Versetzen von je $1,4 \mathrm{ml}$ Cyclohexylsulfaminsäurelösung $(100 \mathrm{mg} / \mathrm{l})$ verschiedener $\mathrm{pH}$-Werte mit jeweils $30 \mu \mathrm{l}-\mathrm{Natriumnitritlösung} \mathrm{(} 100 \mathrm{mg} / \mathrm{l})$. Die Zahlenwerte auf den Kurven geben die pH-Werte der Cyclohexylsulfaminsäurelösungen wieder.

Tab. 2. Verhältnis der Zerfallsgeschwindigkeit zur Bildungsgeschwindigkeit (tg $\alpha /$ Peakhöhe) in Abhängigkeit vom pH-Wert und der Nitritkonzentration. Je $1,4 \mathrm{ml}$ Cyclohexylsulfaminsäure-HCl-Lösung $(100 \mathrm{mg} / \mathrm{l})$ wurden mit 10-60 $\mu 1$ Natriumnitritlösung $(1 \mathrm{~g} / 1)$ versetzt.

\begin{tabular}{lllllll}
\hline pH & \multicolumn{6}{l}{ Natriumnitritlösung $(\mu \mathrm{l})$} \\
& 10 & 20 & 30 & 40 & 50 & 60 \\
\hline \multicolumn{7}{l}{ tg $\alpha$ /Peakhöhe } \\
1,09 & 1,08 & 0,91 & 0,801 & 0,948 & 0,789 & 0,779 \\
1,58 & 0,57 & 0,53 & 0,495 & 0,46 & 0,474 & 0,466 \\
2,18 & 0,37 & 0,31 & 0,287 & 0,263 & 0,24 & 0,237 \\
2,61 & 0,155 & 0,144 & 0,135 & 0,127 & 0,125 & 0,125 \\
2,93 & 0,12 & 0,011 & 0,095 & 0,079 & 0,069 & 0,067 \\
3,30 & 0,0400 & 0,0409 & 0,037 & 0,30 & 0,024 & 0,0235 \\
\hline
\end{tabular}

Um die Zerfallsgeschwindigkeit in Abhängigkeit vom pH-Wert zu ermitteln, wurden die errechneten tg $\alpha$-Werte gegen die entsprechenden $\mathrm{pH}$-Werte graphisch dargestellt (s. Abb. 4). Hieraus läßt sich ableiten, daß das N-Nitrosoderivat bei einem $\mathrm{pH}$-Wert von etwa 4,5 eine relativ hohe Stabilität zeigt.

Die Ümsetzungen nach Tabelle 1 zeigten, daß die Erfassungsgrenze dieser Bestimmungsmethode bei der Umsetzung von Cyclohexylsulfaminsäure in der Konzentration von $6,25 \mathrm{mg} / 1$ liegt. (4. Verdünnung, Molverhältnis von 1/1,11 beim pH 2,61) (s. Abb. 5 und Tab. 2). 


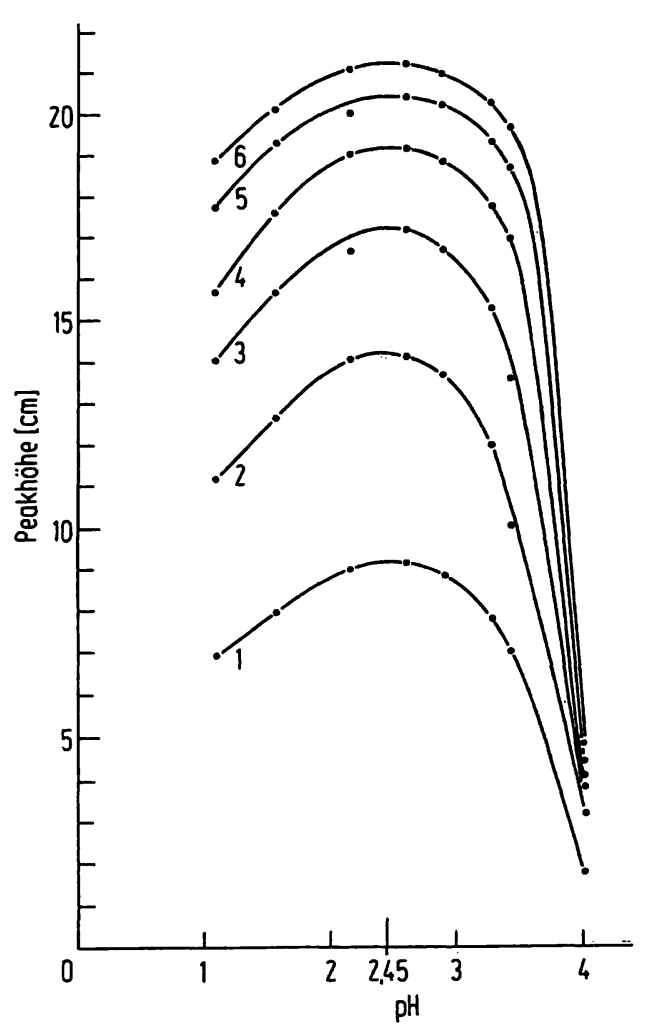

Abb. 3. Maximale Peakhöhe (Absorption) in Abhängigkeit von pH-Wert und Molverhältnis Cyclohexylsulfaminsäure/ Natriumnitrit.

$\begin{array}{lll}\text { Molverhältnis bei Kurve } & (1) & 1 / 0,186 \\ & (2) & 1 / 0,371 \\ (3) & 1 / 0,557 \\ & (4) & 1 / 0,742 \\ (5) & 1 / 0,927 \\ & (6) & 1 / 1,11\end{array}$

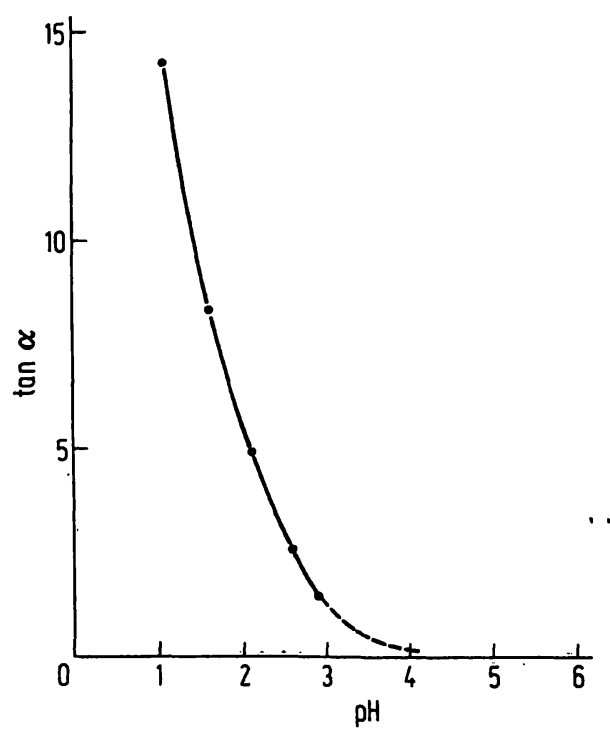

Abb. 4. Abhängigkeit der Zerfallsgeschwindigkeit voọn pH-Wert.

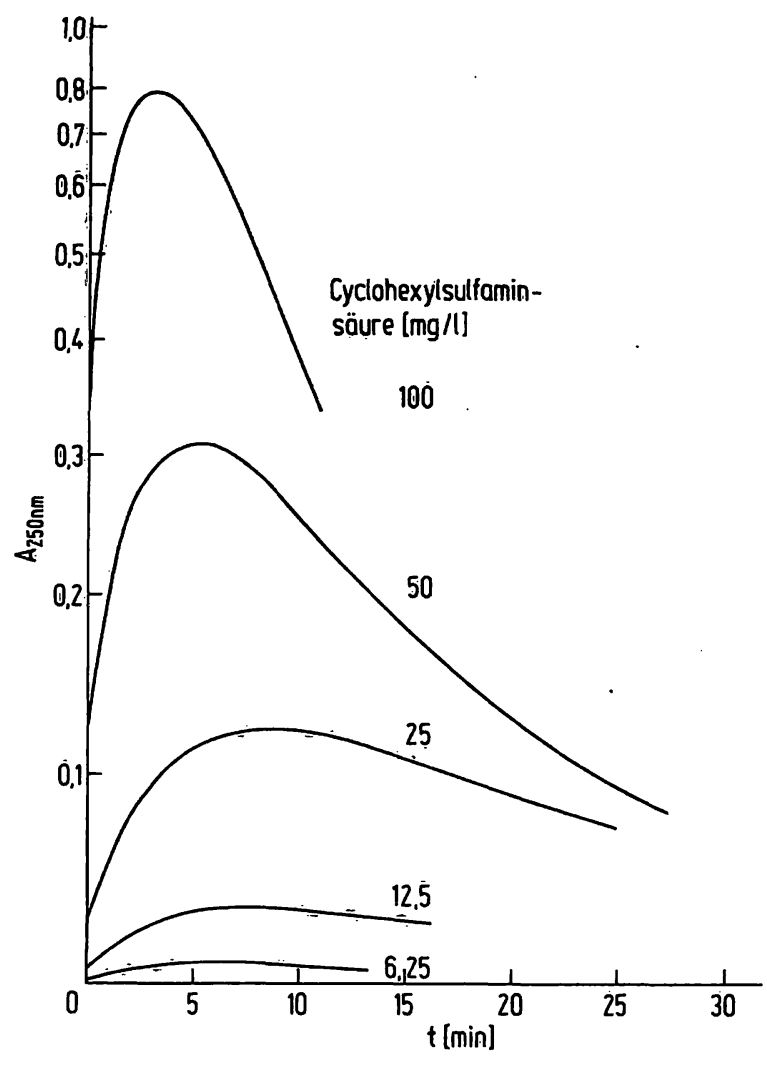

Abb. 5. Änderung der Absorption (bei 250 nm) der Reaktionslösungen nach Versetzen der Cyclohexylsulfaminsäure$\mathrm{HCl}$-Lösungen verschiedener Konzentrationen mit Natriumnitrit im Molverhältnis von 1/1,11. Die Konzentrationsangaben auf den Kurven beziehen sich auf die Cyclohexylsulfaminsäure in der jeweiligen Lösung.

Die Umsetzung in Magensaft und Urin zeigte die gleiche $\mathrm{pH}-\mathrm{Abhängigkeit}$. Während die Magensaftproben und die beiden Urinproben entsprechend ihrer Azidität reagierten, ließß sich die Bildung des N-Nitrosoderivats bei der Umsetzung der Urinprobe mit den pH-Wert 5,1 in Übereinstimmung der Ergebnisse bei der Umsetzung wäßriger Proben gleicher pH-Werte nicht eindeutig nachweisen (s. Abb. 2).

\section{Diskussion}

Die starke pH-Abhängigkeit der Bildungs- als auch der Zerfallsreaktion läßt sich durch Abbildung 2 veranschaulichen. Während im stark sauren Bereich (pH 1,09 und $1,58)$ beide Reaktionen rasch verlaufen, zeigt die Zerfallsreaktion beim pH 2;61 eine Halbwertzeit von 11,8 min.

Nach der gleichen Zeitdauer zerfallen beim $\mathrm{pH} 2,93$ lediglich $25 \%$, während beim pH 3,26 zu diesem Zeitpunkt kein Größenunterschied zwivischen Bildungs- und Zerfallsgeschwindigkeit vorliegt. Die Abnahme des Wertes des Quotienten tg $\alpha /$ Peakhöhe mit Zunahme der Nitritkonzentration (s. Tab. 3) ist auf die Ërhöhung der Reaktionsgeschwindigkeit zurückzuführen. Die Reaktionsgeschwindigkeit erhöht sich jedoch nicht nur auf- 
grund der Erhöhung der Nitritkonzentration, sondern auch wegen Änderung des pH-Wertes während der Umsetzung durch das Zerfallsprodukt, die Schwefelsäure.

Bei der Durchführung der Umsetzung in schwach saurer Lösung verläuft die protonen-katalysierte Umsetzung langsamer, da hier keine Schwefelsäure entsteht, und der pH-Wert der Reaktionslösung verschiebt sich hierbei aufgrund des langsamen Verbrauches der salpetrigen Säure zugunsten der Stabilität des primären Reaktionsproduktes (s. Tab. 3 und Abb. 2).

Unter Berücksichtigung der Ergebnisse dieser Arbeit und der von der WHO tolerierten maximalen Belastung des Organismus mit Nitrit $(0,4-0,8 \mathrm{mg} / \mathrm{kg} \cdot \mathrm{d}$; WHO, Technical Report, 1962) ist es wahrscheinlich, daß Spuren des im Magen gebildeten $\mathbf{N}$-Nitrosoderivats in den stabilisierend wirkenden physiologischen $\mathrm{pH}-\mathrm{Bereich}$ gelangen können. Weiterhin ist es ebenfalls wahrscheinlich, daß die Cyclohexylsulfaminsäure, die im Harn unverändert ausgeschieden wird (22), sich mit dem Nitrit umsetzt, welches bei infektiösen Erkrankungen der Harnwege als Reduktionsprodukt des Nitrats entsteht (23).

\section{Literatur}

1. Schmähl, D. (1972), Medical Tribune 14a, 18.

2. Schmähl, D. \& Krüger, F. W. (1972), Arz̄neim.-Forsch. 22, 999-1000.

3. Price, J. M. Biava, C. G., Oser, B. L., Vogin, E. E., Steinfeld, J. \& Ley, H. L. (1970), Science 167, 1131-1132.

4. Chowaniec, J., Wakefield, J. St. J. \& Hicks, R. M. (1974), Br. J. Cancer 30, 180-181.

5. Leonard, A. \& Linden, G. (1972), C. R. Soc. Biol. (Paris) $166,468-470$.

6. Lorke, D. (1973), Humangenetik 18, 165-170.

7. Bauchinger, M., Schmid, E., Pieper, M. \& Zöllner, N. (1970), Dtsch. Med. Wochenschr. 95, 2220-2223.

8. Green, S., Palmer, K. A. \& Legator, M. S. (1970), Fd. Cosmet. Toxicol. 8, 617-623.

9. Brower, L. P. (1970), Science 170, 558.

10. Bickel, M. H., Burkard, B., Meier-Strasser, E. \& von den Broek-Boot, M. (1974), Xenobiotica 4, 425-439.

11. Lorke, D. \& Machemer, L. (1975), Humangenetik 26, 199-205.

12. Dalderup, L. M. \& Wisser, W. (1971), Experientia 27, 519-521.
Tab. 3. Änderung des pH-Wertes der Reaktionslösung zum Zeitpunkt 30 min nach Reaktionsbeginn. (Molverhältnis: Cyclohexylsulfaminsäure/Nitrit $=1 / 1,11$ ).

\begin{tabular}{ll}
\hline $\begin{array}{l}\text { pH der Cyclohexylsulfamin- } \\
\text { säurelösung }\end{array}$ & $\begin{array}{l}\text { pH der Reaktionslösung } \\
30 \text { min nach Reaktionsbeginn }\end{array}$ \\
\hline 1,09 & 0,93 \\
1,58 & 1,45 \\
2,18 & 1,98 \\
2,61 & 2,51 \\
2,93 & 2,80 \\
3,26 & 3,15 \\
3,30 & 3,20 \\
3,42 & 3,87 \\
4,06 & 4,50 \\
5,10 & 5,85 \\
\hline
\end{tabular}

Eine Aussage über ein etwaiges Risiko bei der Verwendung von Cyclamat als Zuckerersatzmittel muß tierexperimentellen Untersuchungen mit dem N-Nitrosoderivat bzw. Cyclamat/Nitrit vorbehalten bleiben.
13. Beringer, A. (1973), Wien. Med. Wochenschr. 123, 41-48.

14. Kinawi, A., Döring, D. \& Witte, I. (1977), Arzneim.Forschung 27, 747-750.

15. Kinawi, A., Onken, A., Rozyczka, B. \& König, W. (1977), Arzneim.-Forsch. 27, 363-367.

16. Kinawi, A. \& Schuster, T., Arzneim.-Forschung, 28, 219225 (1978).

17. Dalziel, J. A. W., Johnson, R. M. \& Shenton, A. J. (1972), Analyst 97, 719-722.

18. Shenton, A. J. \& Johrison, R. M. (1973), Analyst 98, 749-754.

19. Shenton, A. J. \& Johnson, R. M. (1973), Analyst 98, 745-748.

20. Shenton, A. J. \& Johnson, R. M. (1971), Tetrahedron 27, $1461-1464$.

21. Eisenbrand, G. \& Preussmann, R. (1975), Arzneim.-Forsch. $25,1472-1475$.

22. Renwick, A. G. \& Williams, R. T. (1972), Biochem. J. 192, 869-879.

23. Klinisches Labor, Merck. 11. Auflage (1970), 331-332.
Ass. Prof. Dr. A. Kinawi Arbeitsgruppe Umweltmutagene Ostpreußendamm 111

D-1000 Berlin 45

\section{Preis Biochemische Analytik 1978}

Die Deutsche Gesellschaft für Klinische Chemie verlieh den „Preis Biochemische Analytik 1978“ zu gleichen Teilen an die Biochemiker Prof. Dr. Jerker Porath, Uppsala (Schweden), und Prof. Dr. Shmuel Shaltiel, Rehovot (Israel), für ihren Beitrag zur Verbesserung der Spezifität der chromatographischen Analytik.

Prof. Pöräth hat in früheren Arbeiten bereits entscheidende Grundlagen für die heutigen breiten Anwendungsmöglichkeiten chromatographischer Verfahren in der biochemischen Analytik geschaffen. Der Preis wird Prof. Porath zuerkannt für die Entwicklung zweier neuer chromatographischer Prinzipien: der Metall-Chelat-Affinitätschromatographie und der Charge-Transfer-Chromatographie. Prof. Shaltiel erhält den Preis für seinen grundlegenden Beitrag zur Theorie und Praxis der Hydrophobchromatographie. Prof. Shaltiel hat damit erstmals Wege gewiesen, chromatographische Verfahren auch auf zelluläre Systeme anzuwenden. 